\title{
Tratamiento quirúrgico de fracturas supracondíleas en pacientes pediátricos mediante técnica de fijación externa lateral $\mathrm{AO}$
}

\author{
Surgical treatment of supracondylar fractures in pediatric patients \\ using AO external lateral fixation technique
}

\author{
Segundo-Primero $\mathrm{G},{ }^{*}$ Casas-López $\mathrm{M},{ }^{\ddagger}$ Ruiz-Mejía O,${ }^{\S}$ Tapia-De la O V ${ }^{\S}$
}

Hospital de Traumatología y Ortopedia «Lomas Verdes». Instituto Mexicano del Seguro Social. Estado de México.

RESUMEN. Introducción: Las fracturas del húmero distal representan 70\% de las fracturas en niños menores de 12 años. Es necesario contar con conocimientos sobre este tipo de lesiones, las alternativas terapéuticas existentes y las posibles complicaciones. Objetivo: Valorar los resultados funcionales de la técnica de fijación $\mathrm{AO}$ en el manejo de fracturas supracondíleas humerales en pacientes pediátricos. Material y métodos: Se incluyeron los casos ingresados en el Servicio de Ortopedia Pediátrica intervenidos quirúrgicamente mediante fijación externa método AO, del $1^{\circ}$ de Julio al 31 de Diciembre de 2018. Los datos fueron obtenidos mediante el seguimiento desde el ingreso hasta su alta de la consulta externa, realizando la evaluación clínica funcional mediante la Mayo Elbow Performance Score para evaluar la función del codo. Resultados: Se incluyeron cinco casos con fractura supracondílea humeral tratados mediante fijación externa método AO, con un promedio de edad de seis años. De los cinco casos, tres $(60 \%)$ sufrieron la lesión en miembro superior derecho y dos (40\%) en miembro superior izquierdo, un paciente (20\%) presentó lesión parcial del nervio radial. El puntaje obtenido en la escala Mayo Elbow Performance Score fue de 80 puntos en promedio. Conclusiones: El tratamiento de las fracturas supracondíleas humerales mediante técnica de fijación externa AO muestra resultados buenos, posicionándolo como una opción terapéutica alternativa de tratamiento.
ABSTRACT. Introduction: Fractures of the distal humerus represent $70 \%$ of fractures in children under 12 years. It is necessary to have knowledge about this type of injury, the therapeutic alternatives, and the possible complications. Objective: To assess the functional results of the AO fixation technique in the management of humeral supracondylar fractures in pediatric patients. Material and methods: Patients admitted to the pediatric orthopedics service were surgically treated by external fixation AO method, from July 1 to December 31, 2018. The data were obtained by monitoring from admission of patients until discharge from the outpatient clinic performing the functional clinical evaluation using the Mayo Elbow Performance Score to evaluate the function of the elbow. Results: We included 5 patients with humeral supracondylar fracture treated by external fixation AO method, with an average age of 6 years. Of the 5 cases, $3(60 \%)$ suffered the right upper limb injury and $2(40 \%)$ the upper left limb, 1 patient $(20 \%)$ had partial radial nerve injury. The score obtained on the Mayo Elbow Performance Score was 80 points on average. Conclusions: The functional result of the treatment of humeral supracondylar fractures by means of AO external fixation technique, shows good results, positioning it as an alternative therapeutic treatment option.

\section{Nivel de evidencia: IV}

\footnotetext{
* Médico Residente de 4to año en Ortopedia.

¥ Jefe del Servicio de Ortopedia Pediátrica.

$\S$ Médico adscrito.
}

Unidad Médica de Alta Especialidad en Traumatología y Ortopedia. Hospital de Traumatología y Ortopedia «Lomas Verdes» del Instituto Mexicano del Seguro Social. Estado de México.

Citar como: Segundo-Primero G, Casas-López M, Ruiz-Mejía O, Tapia-De la OV. Tratamiento quirúrgico de fracturas supracondíleas en pacientes pediátricos mediante técnica de fijación externa lateral AO. Acta Ortop Mex. 2020; 34(3): 195-199.

https://dx.doi.org/10.35366/97073

\author{
Dirección para correspondencia: \\ Dr. Gerardo Segundo-Primero \\ Purépechas Núm. 6, edificio C, Dpto. C 104, \\ Col. Pueblo Santa Cruz Acatlán, CP 53150, \\ Naucalpan de Juárez, Estado de México. \\ E-mail: gerardoseg1@hotmail.com
}

www.medigraphic.com/actaortopedica 
Palabras clave: Fracturas, húmero, niños, fijación externa, codo.

\section{Introducción}

Las fracturas del húmero distal representan $70 \%$ de las fracturas entre los cinco a 10 años y corresponden al 30\% de las fracturas de la extremidad torácica en los niños. ${ }^{1}$ En esta etapa de inmadurez esquelética de los niños, es necesario contar con los conocimientos adecuados sobre este tipo de lesiones, las alternativas terapéuticas existentes y las posibles complicaciones de la fractura per se y del tratamiento elegido. ${ }^{2,3,4}$

Dentro de las metas del tratamiento, está el lograr una reducción anatómica y funcional, restituir el ángulo de
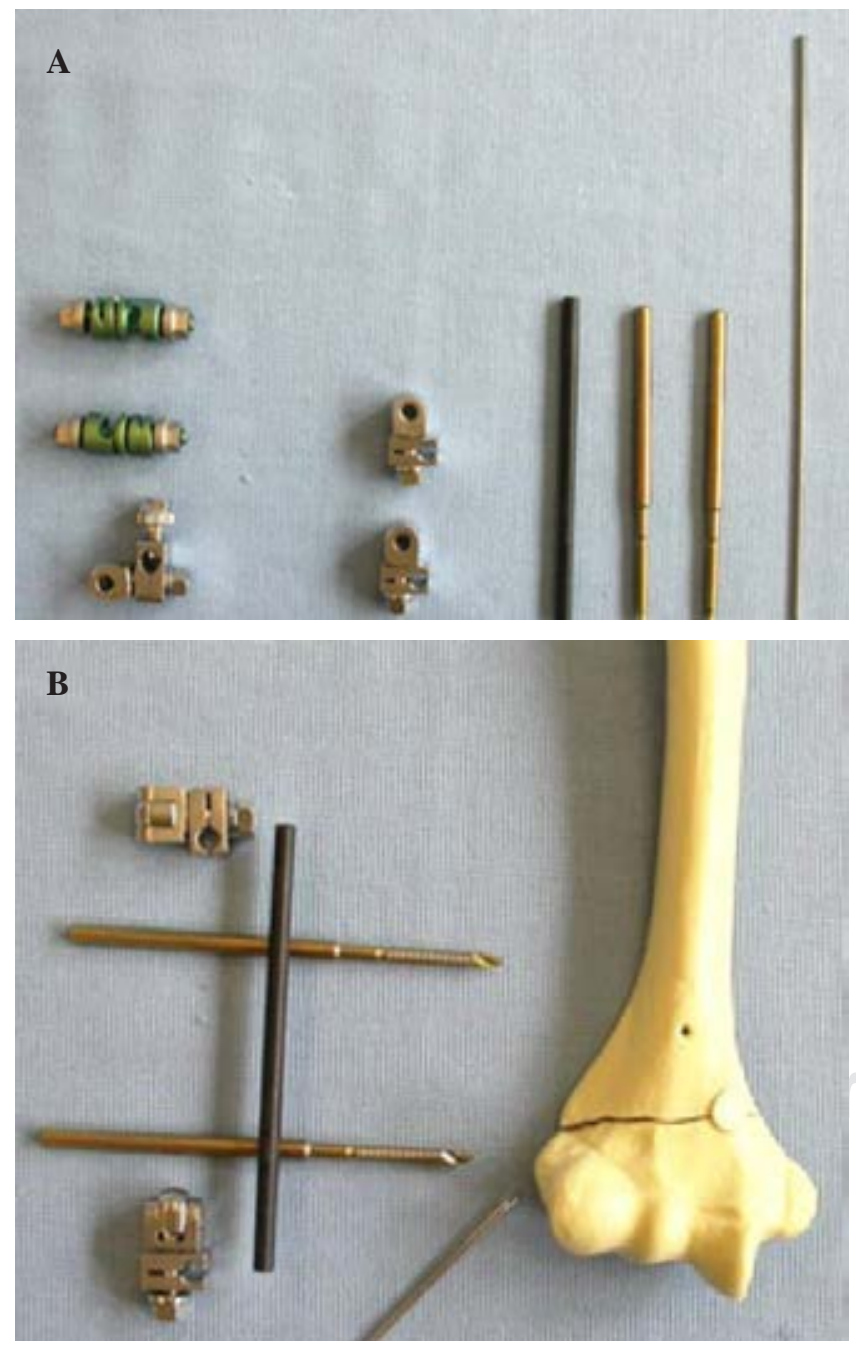

Figura 1: Elementos para la fijación externa lateral de la fractura supracondílea. A) Elementos necesarios para la fijación externa: dos clavos Schanz roscados, dos abrazaderas universales, barra de fibra de carbono. B) Conformación del fijador externo en relación con el húmero.
Keywords: Fractures, humerus, children, external fixation, elbow.

Bowman, evitar lesiones neurovasculares durante el mismo o su manipulación, así como evitar secuelas (como la deformación en varo o valgo, ante y recurvatum), lo que implicaría deformidades funcionales y estéticas. ${ }^{5,6,7}$

El estándar de oro en el tratamiento de las fracturas supracondíleas humerales ha sido el «enclavijamiento percutáneo» con clavillos Kirschner, existiendo varias técnicas y conformaciones de los clavillos descritos en la literatura mundial (clavillos cruzados, cruzados múltiples, paralelos laterales con dos o tres clavillos, etcétera), las cuales buscan brindar mayor estabilidad en la fijación de la fractura, así como también evitar la lesión de estructuras neurológicas, como sucede en 3 y $5 \%$ de los casos con el enclavijamiento medial al comprometer el nervio cubital. 5,6,7,8,9,10

Se ha descrito que el uso de TENS endomedulares por vía retrógrada puede ser eficaz en manos hábiles como método de fijación estable y adecuado, ya que su inserción es en el húmero proximal y requiere mayor destreza. ${ }^{11}$

Por último, también contamos con la posibilidad del uso de fijación externa mediante un marco en $\mathrm{C}$ constituido por dos clavos de Schanz $4.0 \mathrm{~mm}$ de diámetro, $80 \mathrm{~mm}$ de longitud y por lo menos $25 \mathrm{~mm}$ de rosca a $2.5 \mathrm{~mm}$ de diámetro, una barra lateral de $120 \mathrm{~mm}$ de longitud y $4.0 \mathrm{~mm}$ de diámetro y dos rótulas universales a $4.0 \mathrm{~mm}$, complementados con un clavillo Kirschner distal antirrotacional de colocación lateral a nivel del cóndilo humeral de $1.6 \mathrm{~mm}$ de diámetro, siendo este método el propuesto por AO para el manejo de fracturas supracondíleas humerales para niños mayores de tres años (Figura 1). ${ }^{12}$

Entre las ventajas descritas para el método AO se encuentran: posibilidad de poder usar el Schanz distal como joystick durante la reducción del segmento distal del trazo fracturario y posible mayor estabilidad de segmento distal; riesgo mínimo para lesión de nervio cubital; posibilidad de iniciar con movimientos de flexoextensión de manera prematura durante el período de consolidación que va de las cuatro a seis semanas, además de no necesitar férula adicional conservando el movimiento de pronosupinación, pudiendo desarrollar actividades de baja demanda funcional de manera independiente. ${ }^{13}$ La presente investigación tiene como objetivo el de valorar funcional y radiográficamente casos pediátricos con fractura supracondílea humeral tratados de manera quirúrgica mediante la técnica de fijación externa AO.

\section{Material y métodos}

Estudio de serie de casos, de tipo observacional, retrospectivo, transversal y descriptivo. Para su realización 
se incluyeron casos con diagnóstico de fractura supracondílea Gartland III, quienes se trataron de manera quirúrgica con fijación externa mediante el método $\mathrm{AO}$, durante el período comprendido entre el 1 de Julio al 31 de Diciembre de 2018.

\section{Resultados}

Se incluyeron cinco pacientes pediátricos con diagnóstico de fractura supracondílea humeral, manejados con fijación externa técnica AO. Un promedio de edad de 7.8 (de seis a 11 años), todos de sexo masculino. La extremidad dominante fue la más afectada en tres de ellos, los cinco pacientes estudiados son diestros.

Referente al mecanismo de la lesión, la más frecuente fue la caída del plano de sustentación. De la fecha de la lesión a la hospitalización en el servicio, transcurrieron un promedio de 2.4 días y la cirugía fue llevada a cabo en un promedio de 6.4 días después de la lesión. El egreso tuvo un promedio de 41.6 horas postoperatorias. En nuestro grupo de estudio, se mantuvo una férula antiálgica por espacio de los primeros 15 días después de la cirugía.

Después del egreso, la primera consulta se llevó a cabo en un promedio de 18 días postoperado, en ella se retiró la férula y se enseñó al familiar encargado los cuidados correspondientes del fijador. En esta primera consulta, la evaluación del dolor arrojó un promedio en la escala visual analógica (EVA) de 9 puntos y la evaluación del arco de movimiento estableció un rango de 19 grados.

En una segunda evaluación a los 57 días en promedio, el puntaje obtenido en la escala EVA fue de 5.6 puntos en promedio, con un rango de movilidad de 40 grados antes del retiro del fijador con un aumento en el arco de movilidad de $100 \%$ y una disminución del dolor de la misma magnitud con respecto a la consulta previa.

La evaluación final a los 115 días de la cirugía arroja un EVA de 2 puntos en promedio y un arco de movimiento de 131 grados, lo que permitió establecer una puntuación total de Mayo Elbow Performance Score (MEPS) de 80 promedio, siendo el más bajo de 65 puntos y la más alta de 95 (Tabla 1).

El tiempo quirúrgico mostró un promedio de 46 minutos con un rango de 30 a 70 minutos. Como complicaciones prematuras se detectó una neuropraxia del nervio radial $\mathrm{y}$, dentro de las secuelas postoperatorias mediatas, se evidenció traslación posterior del segmento distal en un caso

\begin{tabular}{|crcc|}
\hline \multicolumn{4}{c}{$\begin{array}{c}\text { Tabla 1: Resultados finales del paciente escala } \\
\text { MEPS (Mayo Elbow Performance Score). }\end{array}$} \\
\hline \multirow{2}{*}{ Paciente } & Edad & Total MEPS pts. & Resultado \\
\hline 1 & 7 & 80 & Bueno \\
2 & 8 & 65 & Regular \\
3 & 7 & 95 & Excelente \\
4 & 11 & 80 & Bueno \\
5 & 6 & 80 & Bueno \\
\hline
\end{tabular}
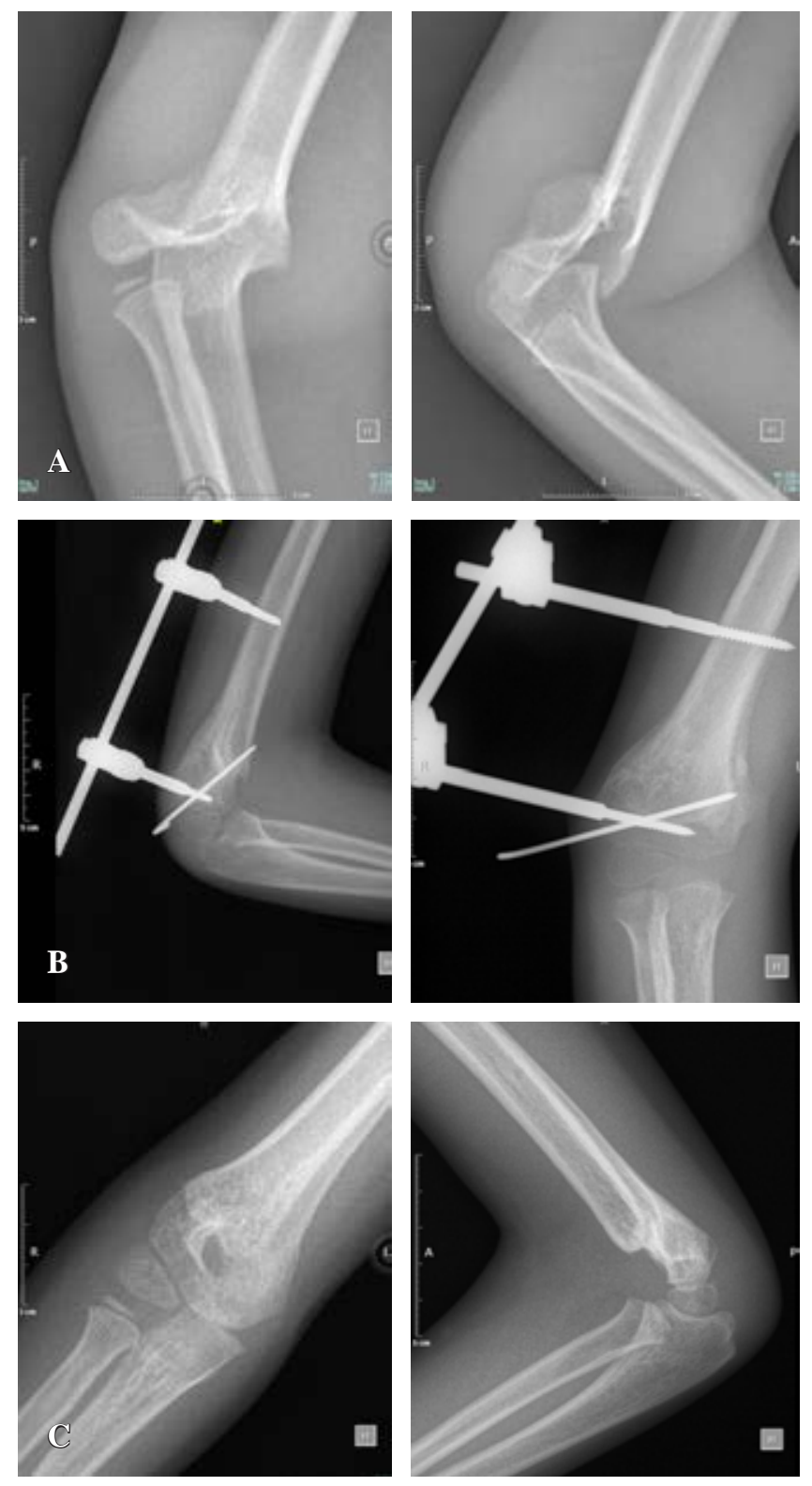

Figura 2: Radiografías en proyección anteroposterior y lateral de codo. A) Preoperatorias. B) Postoperatorias donde se observa la fijación externa y el clavillo. C) Controles finales sin el fijador externo donde se observa un ligero desplazamiento hacia posterior del fragmento distal.

(Figura 2). En relación con las deformidades angulares, se evidenció una deformidad en varo en tres casos $(60 \%)$ con un máximo de 4 grados de la media y dos en valgo con un máximo de 6 grados.

\section{Discusión}

Los resultados obtenidos muestran que la presentación de esta patología es mayor en la población masculina, la fractura principal del codo pediátrico es la principal como lo documenta la literatura mundial. El grupo de edad afectado corresponde a los escolares y adolescentes de entre seis y 10 años. 
La clasificación de Gartland fue la utilizada, la cual mostró a la fractura tipo IIIB (que implica la fractura supracondílea con desplazamiento posterolateral del codo) como la más reportada. Todos los casos se clasificaron como tipo III con afectación predominante de la extremidad derecha en caso de que el sujeto tuviera dominancia derecha, interpretándose como un mecanismo de protección del brazo dominante.

Se reporta que el mecanismo de lesión de ésta es la carga axial con codo en extensión, presentándose en la totalidad de los casos, misma que coindice con la literatura internacional y cuyo evento se refleja en caídas del plano de sustentación en $60 \%$, seguida de lesiones en actividades recreativas.
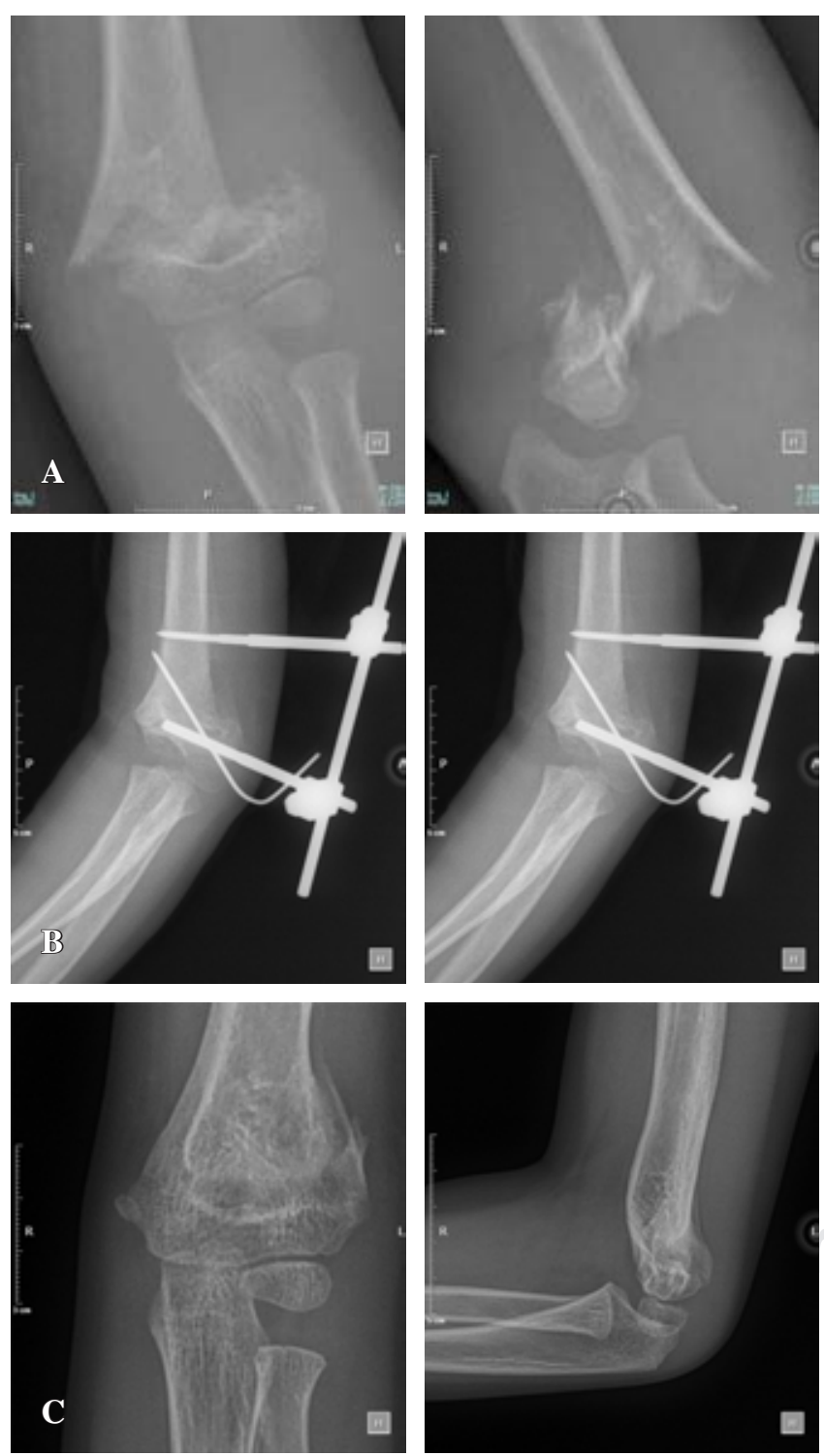

Figura 3: Radiografías en proyección anteroposterior y lateral de codo. A) Preoperatorias donde se observa el desplazamiento del fragmento distal hacia lateral y posterior. B) Postoperatorias, se observa la alineación y la presencia del fijador externo lateral con el clavillo antirrotacional. C) Finales que muestran consolidación ósea y sin el sistema de fijación.
Tabla 2: Escala MEPS (Mayo Elbow Performance Score). ${ }^{14,15,16}$

Puntos

1) Dolor (máximo 45 puntos)

Ninguno

Ligero 45

Moderado

30

Severo

15

2) Arco de movilidad (máximo 20 puntos): flexión normal $\left(140^{\circ}\right)$

$>100^{\circ}$

$50-100^{\circ} \quad 15$

$<50^{\circ}$

3) Estabilidad (máximo 10 puntos)

Estable

Moderada inestabilidad

Gran inestabilidad

5

4) Actividad sin ayuda (máximo 25 puntos)

Posibilidad de peinarse 5

Comer sin ayuda 5

Higiene personal 5

Vestirse 5

Calzarse

Suma total (máximo 100 puntos) Suma paciente

Resultados: Malo $=$ menos de 60, Regular $=60-74$, Buena $=75-89$,

Excelente $=90-100$.

La indicación quirúrgica del caso estuvo determinada por la clasificación de Gartland, por imagen radiográfica (Figura 3). En nuestra serie de casos, el tratamiento empleado fue reducción abierta en dos casos y cerrada en tres casos, los cuales se estabilizaron mediante fijación externa lateral método AO.

La utilización del MEPS (Tabla 2) arrojó las puntuaciones comentadas, encasillando a la totalidad de los casos con resultados buenos, el paciente que presentó lesión parcial del nervio radial al finalizar el estudio mostró recuperación total del mismo, sin secuelas limitantes para la función.

El resultado funcional del codo después de este tipo de lesión, manejado con el método de fijación externa lateral, fue bueno en más de $80 \%$ de los casos, en parte al diagnóstico y a la resolución quirúrgica temprana de la lesión, así como al protocolo de seguimiento ambulatorio correspondiente llevado hasta el alta del caso (Tabla 1).

Nuestra serie de casos es muy pequeña y no permite obtener conclusiones sobre los resultados, o si muestra que es una técnica reproducible y no libre de complicaciones. Aunque se documenta un resultado funcional bueno de acuerdo con la escala MEPS para el tratamiento de las fracturas supracondíleas, sólo se incluye un seguimiento de cuatro meses en promedio. La fijación externa lateral propuesta por AO debe ser empleada en pacientes con fracturas supracondíleas, se requiere de características precisas para las cuales está diseñado este tipo de tratamiento, además de ser necesario ejecutar la técnica de la mano de un cirujano familiarizado y entrenado con la técnica específica. 
Bibliografía

1. Scaglione M, Giovannelli D, Fabbri L, Dell'omo D, Goffi A, Guido G. Supracondylar humerus fractures in children treated with closed reduction and percutaneous pinning. Musculoskelet Surg. 2012; 96(2): 111-6.

2. Chaturvedi H, Khanna V, Bhargava R, Vaishya R. Functional outcomes in paediatric supracondylar fracture humerus treated with percutaneous pinning with cross K-wires. Apollo Medicine. 2017; 14: 34-41.

3. Meza SJF. Fractura supracondílea humeral en niños. Ortho-tips. 2009; 5(3): 218-25.

4. Palmer EE, Niemann KM, Vesely D, Armstrong JH. Supracondylar fracture of the humerus in children. JBJS. 1978; 60(5): 653-6.

5. Zorrilla S de Neira J, Prada-Cañizares A, Marti-Ciruelos R, PretellMazzini J. Supracondylar humeral fractures in children: current concepts for management and prognosis. Int Orthop. 2015; 39(11): 2287-96.

6. Reisoglu A, Kazimoglu C, Hanay E, Agus H. Is pin configuration the only factor causing loss of reduction in the management of pediatric type III supracondylar fractures? Acta Orthop Traumatol Turc. 2017; 51(1): 34-8.

7. Gil Albarova J, Herrera A. Revisión y actualización del tratamiento de las fracturas supracondíleas de húmero en la infancia. Rev Esp Cir Osteoartic. 2013; 255(48): 110-22.

8. Millis MB, Singer IJ, Hall JE. Supracondylar fracture of the humerus in children. Further experience with a study in orthopaedic decisionmaking. Clin Orthop Relat Res. 1984; (188): 90-7.
9. Li J, Fu D, Yu C, Wang S, Ze R, Tang X. Surgical management of delayed irreducible Gartland III supracondylar fractures in children: open reduction and internal fixation versus external fixation. $J$ Shoulder Elbow Surg. 2017; 26(2): 299-304.

10. Silva M, Kazantsev M, Aceves Martin B, Delfosse EM. Pediatric supracondylar humerus fractures: is surgeon experience a surrogate for the need of open reduction? J Pediatr Orthop B. 2018; 27(2): 103-7.

11. Pesenti S, Ecalle A, Gaubert L, Peltier E, Choufani E, Viehweger E, et al. Operative management of supracondylar humeral fractures in children: Comparison of five fixation methods. Orthop Traumatol Surg Res. 2017; 103(5): 771-5.

12. Slongo T. Radialer externer Fixateur zur geschlossenen Behandlung problematischer suprakondylärer Humerusfrakturen Typ III und IV bei Kindern und Jugendlichen. Eine neue chirurgische Technik. Oper Orthop Traumatol. 2014; 26(1): 75-96; quiz 97.

13. Bogdan A, Quintin J, Schuind F. Treatment of displaced supracondylar humeral fractures in children by humero-ulnar external fixation. Int Orthop. 2016; 40(11): 2409-15.

14. Turchin DC, Beaton DE, Richards RR. Validity of observer-based aggregate scoring systems as descriptors of elbow pain, function, and disability. J Bone Joint Surg Am. 1998; 80(2): 154-62.

15. Longo UG, Franceschi F, Loppini M, Maffulli N, Denaro V. Rating systems for evaluation of the elbow. Br Med Bull. 2008; 87: 131-61.

16. Min W, Ding BC, Tejwani NC. Comparative functional outcome of AO/OTA type $\mathrm{C}$ distal humerus fractures: open injuries do worse than closed fractures. J Trauma Acute Care Surg. 2012; 72(2): E27-32. 